\title{
Efficacy and safety of mitoxantrone, as an initial therapy, in multiple sclerosis: Experience in an Indian tertiary care setting
}

\author{
B. S. Singhal, Sheth Geeta, Shilpa G. Hundalani, Suresh Menon \\ Department of Neurology, Bombay Hospital Institute of Medical Sciences, India
}

\author{
Address for correspondence: \\ Dr. B. S. Singhal, \\ Bombay Hospital Institute of \\ Medical Sciences, MRC Room \\ No. 131, 12, New Marine Lines, \\ Mumbai - 400 020, India. \\ E-mail: bssinghal@gmail.com
}

\begin{abstract}
Background and Purpose: Mitoxantrone is an approved disease modifying agent for treatment of multiple sclerosis (MS). The aim of the study was to assess its efficacy and safety in Indian MS patients. Materials and Methods: A total of 23 patients with clinically definite MS (Poser criteria) were enrolled in an open label study. Of which, 21 satisfied the McDonald's criteria for MS and two satisfied the diagnostic criteria of neuromyelitis optica (NMO). The numbers of relapses and expanded disability status scale (EDSS) score were used as primary and secondary outcome measures. The patients were monitored for the adverse effects. Results: In 17 (15 MS and two NMO) patients who completed one year of therapy, there was significant difference in the mean annual relapse rates [before $0.879 \pm 0.58$; on mitoxantrone $0.091 \pm 0.17,(P=0.003)]$. Of the 17 patients, ten (MS 9 and NMO I) completed therapy for two years. Annual relapse rates [before (1.024 \pm 0.59$)$, on therapy (0.155 \pm 0.21$),(P=0.0054)]$ and EDSS score [before start of therapy 5.3, at the end of therapy $2.4,(P=0.001)]$ showed significant benefit in the ten patients who completed two years therapy. This benefit persisted during the mean follow-up period of two and a half years after completion of therapy. The adverse events noted in the entire cohort were leucopenia in four patients and asymptomatic reversible decrease in cardiac ejection fraction in one patient. Leucopenia was severe in two patients requiring discontinuation of the therapy and mitoxantrone was also discontinued in the patient with cardiotoxicity. Conclusions: Mitoxantrone, as an initial therapy, decreases clinical exacerbations and disability progression, and has a reasonable safety profile in Indian patients with MS and NMO.
\end{abstract}

Key words: Mitoxantrone, multiple sclerosis, novantrone

\section{Introduction}

Multiple sclerosis (MS) is an inflammatory demyelinating disease of the central nervous system (CNS) with a variable course. Four clinical patterns have been defined by international consensus: Relapsing remitting (RR), secondary progressive (SP), primary progressive (PP), and progressive relapsing (PR) ${ }^{[1]}$ Most patients with MS eventually develop significant disability affecting their quality of life. Mitoxantrone (Novantrone), a synthetic anthracenedione derivative, is an antineoplastic, immunomodulatory agent that is shown to delay the progression of disability and decrease the frequency of relapses in patients with MS. ${ }^{[2-4]}$ The associated adverse effects are usually of mild to moderate severity, although high doses have potential to cause cardiotoxicity. ${ }^{[5]}$ There is also a concern regarding therapy-related acute leukemia (TRAL). Ellis and Boggild ${ }^{[6]}$ reported TRAL in $0.30 \%$ of MS patients treated with mitoxantrone. Martinelli ${ }^{[7]}$ reported an incidence of $0.74 \%$ in a large cohort from Italy. In India, cost of medication is an important limiting factor in the treatment of MS patients. As mitoxantrone is a much cheaper drug in India compared to other disease modifying agents such as 
interferons and glatiramer acetate (GA), it was offered as first line treatment to patients with MS. We present our experience with the use of mitoxantrone as an initial therapy in Indian patients with MS.

\section{Materials and Methods}

Patients were recruited from a cohort of MS patients attending the neurology clinic in a large tertiary care hospital between 2002 and 2007. The treatment protocol was approved by the Institutional Ethics Committee. To be eligible for therapy, patients had to satisfy the following criteria: (a) clinically definite MS according to the Poser criteria ${ }^{[8]}$ with RR, SP, or PR pattern; (b) no previous treatment with any disease modifying therapy. Patients with PPMS, cardiac dysfunction with reduced ejection fraction (EF), hepatic, or renal disease were excluded. Other exclusion criteria were: Abnormal baseline white blood cells $\left(<4 \times 10^{9} / 1\right.$, neutrophils $<2 \times 10^{9} / 1$, platelets $\left.<100 \times 10^{9} / 1\right)$; pregnant or lactating women; and those in reproductive age group not willing to use contraception. It was an open label study and the examining physicians were not blinded. The potential benefits and risks were explained in detail and written informed consent was obtained prior to the initiation of therapy.

Patients were admitted for slow intravenous infusions of $12 \mathrm{mg} / \mathrm{m}^{2}$ of mitoxantrone at three-month intervals (up to a maximum cumulative dose of $140 \mathrm{mg} / \mathrm{m}^{2}$ ). An intravenous antiemetic (granisetron) was given prophylactically to all the patients prior to administering mitoxantrone to prevent drug induced nausea and vomiting.

Interval history, physical examination, and neurological examination including the EDSS ${ }^{[9]}$ were recorded at every visit. The patients were monitored for adverse reactions. Hemoglobin, WBC, platelet count, SGPT, electrocardiogram, 2D echo, and chest X-ray were performed prior to every three month infusion. Blood counts and SGPT were repeated one week after each dose and at two weekly intervals thereafter while the patient was on mitoxantrone, and at six-monthly intervals after therapy was completed. If a patient had any abnormal blood parameter, they were monitored carefully and restarted on a lower dose of mitoxantrone only after complete normalization of blood parameters.

Patients who experienced a relapse (defined as the occurrence of new neurological symptoms with new focal neurological deficits lasting at least 48 hours, and preceded by stability or improvement for at least 30 days) were treated with a three to five day course of intravenous methylprednisolone (1000 mg/day). In order to assess clinical outcome, we compared the number of relapses per year from the clinical history and medical records before and on mitoxantrone treatment (Wilcoxon signed rank test). The EDSS scores were evaluated before and after mitoxantrone therapy (baseline or at the time of relapse) and were compared (paired student's $t$-test) as a secondary outcome measure.

\section{Results}

A total of 23 patients (18 female, 5 male) with clinically definite MS (Poser criteria) met the inclusion/exclusion criteria and consented to therapy. Eleven patients had worsening RR pattern, eight patients had SP pattern, and four patients had PR pattern of disease. The mean duration of MS prior to mitoxantrone therapy was 6.65 years. Incorporating the MRI data, 21 patients satisfied the 2005 revised McDonald's criteria of MS.[10,11] Two patients satisfied the diagnosis of Neuromyelitis Optica (NMO) according to the revised diagnostic criteria by Wingerchuk, ${ }^{[12]}$ although the serum NMO antibody test was not done.

Figure 1 shows the duration of treatment and follow-up for all patients. Six patients discontinued therapy after receiving only one to three doses of therapy. One patient developed severe leucopenia requiring granulocyte-colony stimulating factor (GCSF) therapy after first dose; one patient had a severe MS relapse after the second dose and discontinued treatment; three patients withdrew since they lived out of town and did not have access to medical facilities locally; and one patient was lost to follow-up.

A total of 17 patients completed one year of therapy with a mean of four doses in that period. The mean annual relapse rates before and on mitoxantrone therapy in this group were $0.879 \pm 0.58$ and $0.091 \pm 0.17$, respectively, and the difference was statistically significant $(P=0.003)$.

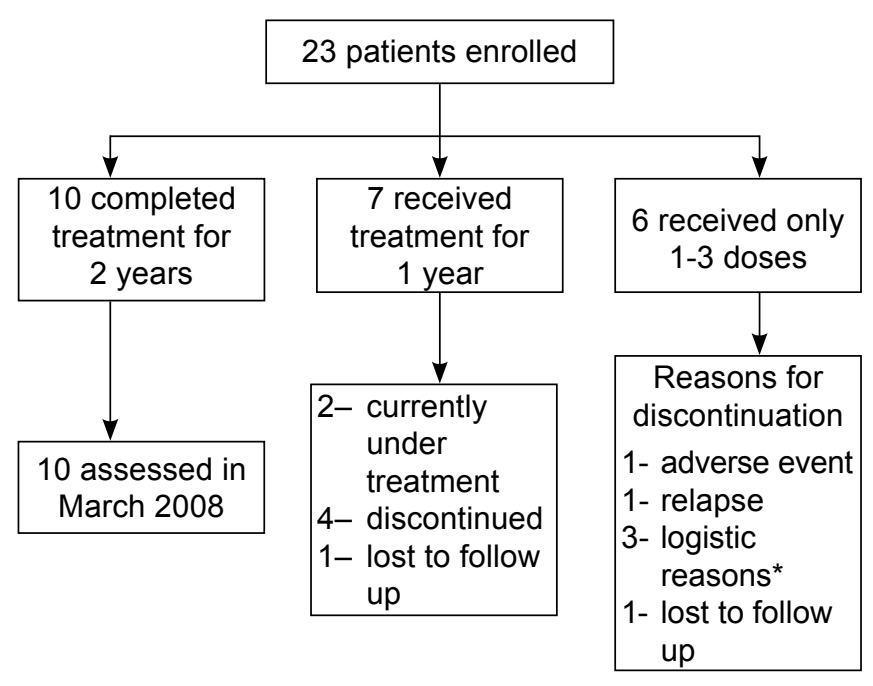

* These patients were referred from distant areas and did not have access to medical facilities to continue treatment in their hometowns.

Figure 1: Study profile 
Of these, ten patients (nine MS and one NMO) completed therapy for two years [Table 1], a mean of eight doses of therapy. The mean annual relapse rate before the onset of therapy was $1.024 \pm 0.59$. The mean annual relapse rate/year during and after mitoxantrone therapy till the last follow-up in March 2008 was $0.155 \pm 0.21$, which was significantly lower than that prior to initiation of mitoxantrone $(P=0.0054)$. The mean EDSS score before starting treatment was 5.3 (range, 2.5-8.5) and at the end of treatment was 2.4 (range, 1-6). The EDSS score of these patients after treatment was significantly better than their EDSS score at the beginning of treatment $(P=0.001)$. The mean duration of follow-up after completion of therapy was 2.5 years (range, four months to four years), and the mean EDSS score at the last follow-up in March 2008 was 2.75 (range, 0-6.5). None of these ten patients had serious adverse effects. None of the patients received another disease modifying agent after discontinuation of mitoxantrone. Three patients had one relapse each while on mitoxantrone therapy. One patient reported two relapses one year after completion of therapy. This patient's EDSS scale score worsened from 1.5 to 6.5 during the total follow-up period of four years after completion of mitoxantrone therapy.

Seven patients (six MS, one NMO) received mitoxantrone for one year only [Table 2], with an average of four doses of therapy. The mean EDSS before starting treatment was 5.1 (range, 2.5-6.5) and at the time of last dose was
3.4 (range, 0-6). The EDSS of these patients at the end of one year was better, though not significantly different from the EDSS at the start of treatment $(P=0.059)$. The mean duration of follow-up was 26 months (range, six months to four years). Of the seven patients who took treatment only for one year, two stopped therapy due to adverse events (significant leucopenia after the fourth dose, decreased EF after the sixth dose); two patients declined further treatment for fear of possible toxicity, one patient was lost to follow-up, and two patients are still receiving treatment.

The study included two patients with relapsing NMO. Of these, one patient has completed two years of treatment and one patient has had one year of treatment. Both these patients showed significant improvement in EDSS while on mitoxantrone. None of them have had any relapses while on treatment. One patient is still undergoing treatment. The other patient who completed treatment for two years has not had any relapse during the follow-up period of two years after treatment completion.

The most notable adverse event in the entire cohort was leucopenia (four patients). In two patients, the leucopenia was severe (one required G-CSF therapy) and occurred after the first and fourth dose, respectively, requiring discontinuation of therapy. The other two patients had mild, transient leucopenia. One patient discontinued therapy after 2D echocardiogram (2D-Echo) showed left ventricular hypokinesia and reduced left ventricular

\begin{tabular}{|c|c|c|c|c|c|c|c|c|c|c|}
\hline $\begin{array}{l}\text { Patient } \\
\text { initials }\end{array}$ & Sex & $\begin{array}{l}\text { Age at } \\
\text { onset } \\
\text { (years) }\end{array}$ & $\begin{array}{l}\text { Disease } \\
\text { course }\end{array}$ & $\begin{array}{l}\text { Duration of } \\
\text { illness before } \\
\text { MTX (years) }\end{array}$ & $\begin{array}{c}\text { Relapses/ } \\
\text { year before } \\
\text { MTX }\end{array}$ & $\begin{array}{l}\text { EDSS } \\
\text { before } \\
\text { MTX }\end{array}$ & $\begin{array}{l}\text { Relapses/year } \\
\text { after starting MTX } \\
\text { till last follow up }\end{array}$ & $\begin{array}{l}\text { EDSS at } \\
\text { last dose } \\
\text { of MTX }\end{array}$ & $\begin{array}{c}\text { Follow up in yrs } \\
\text { after completion } \\
\text { of therapy }\end{array}$ & $\begin{array}{l}\text { EDSS } \\
\text { at last } \\
\text { follow up }\end{array}$ \\
\hline PS & $\mathrm{F}$ & 22 & SP & 16 & 0.31 & 6 & 0 & 4 & 4 & 4 \\
\hline MS & $\mathrm{F}$ & 31 & SP & 6 & 1.16 & 6.5 & 0 & 6 & 4 & 6 \\
\hline $\mathrm{PH}$ & $\mathrm{F}$ & 30 & SP & 2 & 1 & 6 & 0 & 5 & 2 & 5 \\
\hline PA & $\mathrm{F}$ & 35 & PR & 10 & 0.4 & 4.5 & 0.36 & 1.5 & 4 & 6.5 \\
\hline SV & $\mathrm{F}$ & 27 & $\mathrm{RR}$ & 4 & 0.75 & 6 & 0 & 2 & 3 & 2 \\
\hline $\mathrm{NL}$ & $\mathrm{F}$ & 24 & $\mathrm{RR}$ & 5 & 1.25 & 2.5 & 0 & 0 & 2.5 & 0 \\
\hline SJ & $\mathrm{F}$ & 31 & RR & 1 & 2 & 4.5 & 0.29 & 2.5 & 2 & 2 \\
\hline SN & $\mathrm{F}$ & 32 & $\mathrm{RR}$ & 4 & 1 & 5.5 & 0.4 & 1 & 3 & 0 \\
\hline$J N$ & $\mathrm{~F}$ & 30 & $\mathrm{RR}$ & 3 & 1.67 & 3 & 0.5 & 1 & 4 (months) & 1 \\
\hline $\mathrm{RK}^{*}$ & $\mathrm{~F}$ & 34 & $\mathrm{RR}$ & 10 & 0.7 & 8.5 & 0 & 1 & 2 & 1 \\
\hline
\end{tabular}

*Patient with neuromyelitis optica, MTX - Mitoxantrone

\begin{tabular}{|c|c|c|c|c|c|c|c|c|}
\hline $\begin{array}{l}\text { Patient } \\
\text { initials }\end{array}$ & Sex & $\begin{array}{c}\text { Age at onset } \\
\text { (years) }\end{array}$ & $\begin{array}{l}\text { Disease } \\
\text { course }\end{array}$ & $\begin{array}{l}\text { Duration of illness } \\
\text { before MTX (years) }\end{array}$ & $\begin{array}{c}\text { Relapses/year } \\
\text { before MTX }\end{array}$ & $\begin{array}{c}\text { EDSS before } \\
\text { MTX }\end{array}$ & $\begin{array}{c}\text { Relapses/year } \\
\text { after starting MTX }\end{array}$ & $\begin{array}{l}\text { EDSS at last } \\
\text { dose of MTX }\end{array}$ \\
\hline נJ & $\mathrm{F}$ & 26 & $S P$ & 4 & 0.75 & 6.5 & 0 & 6 \\
\hline PS & $M$ & 31 & $\mathrm{SP}$ & 6 & 0.33 & 4.5 & 0 & 4.5 \\
\hline GM & $M$ & 38 & SP & 3 & 0.33 & 5 & 0 & 4.5 \\
\hline AM & $\mathrm{F}$ & 33 & PR & 3 & 0.67 & 6.5 & 0 & 6 \\
\hline $\mathrm{ML}$ & $F$ & 45 & $\mathrm{RR}$ & 1 & 2 & 2.5 & 0 & 0 \\
\hline GK & $\mathrm{F}$ & 52 & $\mathrm{RR}$ & 4 & 0.5 & 4 & 0 & 1.5 \\
\hline$S J^{*}$ & $F$ & 13 & $\mathrm{RR}$ & 16 & 0.13 & 6.5 & 0 & 1 \\
\hline
\end{tabular}

*Patient with neuromyelitis optica, MTX - Mitoxantrone 
ejection fraction (LVEF) of $40 \%$ after a cumulative dose of $48 \mathrm{mg} / \mathrm{m}^{2}$. A follow-up 2D-Echo done three months later showed improvement of the LVEF to $55 \%$. No patient experienced signs of congestive cardiac failure or other clinically significant cardiac dysfunction. Other adverse events included mild hair loss (five patients), urinary tract infection (two patients), and transient mild elevation of SGPT levels (two patients). There was no evidence of mitoxantrone-related leukemia or any other malignancy during follow-up until March 2008.

\section{Discussion}

MS is a common cause of neurological disability in young adults and exhibits considerable clinical, pathologic and radiological heterogeneity. The widespread availability of MRI has led to an increased recognition of MS in India. Early identification of patients is essential because there is evidence that early treatment might delay or limit long-term disability. Interferon $\beta-1 b$, Interferon $\beta-1 a, G A$, mitoxantrone, and natalizumab are disease-modifying agents (DMA) approved by the US Food and Drug Administration (FDA) and the regulatory authorities of many other countries for the treatment of MS.

Mitoxantrone, an intravenously administered anthracenedione antineoplastic agent, is indicated for reducing neurological disability and relapse frequency in patients with secondary progressive multiple sclerosis (SPMS), progressive relapsing multiple sclerosis (PRMS), or worsening relapsing-remitting MS (RRMS). ${ }^{[2-4]}$ There is no evidence of efficacy of mitoxantrone in PPMS or in later stages of SPMS beyond an EDSS score of six. ${ }^{[2]}$ The beneficial effects of mitoxantrone in MS are due to its immunosuppressive action based on nonspecific cytotoxic effects on B and T lymphocytes, and induction of programmed cell death of professional antigen presenting cells, such as dendritic cells. ${ }^{[13]}$ In addition, mitoxantrone may inhibit the migration of inflammatory cells into and within the CNS, thus interfering with one of the key events in the immunopathogenesis of MS. ${ }^{[14]}$ Mitoxantrone also has immunomodulatory properties by decreasing selectively the secretion of cytokines as interferon (IFN)- $\gamma$, tumor necrosis factor- $\alpha$, and IL-2. ${ }^{[15]}$

Although mitoxantrone is not without risk, the potential benefits of reduction in disease progression outweigh the risks in patients with worsening disease. ${ }^{[16]}$ Mitoxantrone has been shown to be a more cost-saving treatment for patients with SPMS or progressive relapsing MS than Interferon $B-1 b{ }^{[17]}$ For Indian patients, the cost of treatment remains a major limiting factor in the use of disease modifying therapy. Although there is hesitation in the use of mitoxantrone due to its potential toxicity, we offered it to patients with MS as first line of therapy due to its relatively low cost compared to interferons or GA.
There have been several studies supporting the use of mitoxantrone in MS. In a double-blind, monotherapy trial [mitoxantrone in multiple sclerosis (MIMS) trial], 194 patients with worsening RRMS or SPMS who received mitoxantrone $12 \mathrm{mg} / \mathrm{m}^{2}$ every three months for two years had significantly fewer relapses $(P<0.001)$ and significantly less deterioration in disability, as measured by change in EDSS score compared with those who received placebo. ${ }^{[2]}$ In a longitudinal open-label prospective study, there was a delayed beneficial effect after mitoxantrone treatment was completed with only a minority of patients showing disability progression once the drug was discontinued. ${ }^{[18]}$ In a retrospective analysis of 304 patients with active relapsing-remitting (RR) or progressive multiple sclerosis (PMS) who were treated with mitoxantrone for two years and followed up for three years thereafter it was found that mitoxantrone treatment induces stable disease for up to two years after discontinuation of mitoxantrone therapy. ${ }^{[19]}$

The use of mitoxantrone has been studied in a diverse patient population. An Iranian study showed mitoxantrone was generally well tolerated and reduced progression of disability and clinical exacerbation in MS patients. ${ }^{[20]}$ In a small Japanese study, the seven patients who continued mitoxantrone therapy for more than three times significantly decreased their relapse rate and EDSS deterioration. ${ }^{[21]}$ In addition to MS, mitoxantrone is also being used for patients with NMO. A study from New York of five patients with relapsing NMO showed clinical improvement in four patients with relapsing NMO. ${ }^{[22]}$

Mitoxantrone has been found to be useful as an induction therapy followed by the use of DMA such as GA. In the study by Vollmer et al. the use of mitoxantrone every month for three months followed by GA reduced the mean relapse rates at 15 months to $0.16 \pm 0.34$ in comparison to $0.32 \pm 0.66$ when GA alone was used. ${ }^{[23]}$ In another study by Ramtahal et al., the average annualized relapse rate was reduced $96 \%$ following mitoxantrone therapy (from 2.7 in the two years before the study to 0.106; $P<0.001) .{ }^{[24]}$ In our study the use of mitoxantrone alone reduced the annualized relapse rates by $89.6 \%$.

In our study of Indian patients, a detailed analysis of the 17 patients who completed either the full course or at least a year of therapy suggests that mitoxantrone may be an effective treatment option. Ten patients who completed treatment for two years showed a nearly three point mean improvement in EDSS, with only three relapses occurring during this time frame. Nine of these ten showed persistent benefit, and only one patient developed relapses and showed worsening of EDSS after therapy completion. The seven patients who completed at least a year of therapy showed no EDSS progression or relapse while on treatment. Follow up of four of these patients 
for a mean of 2.2 years also revealed no relapses or EDSS progression. The study period in most trials extends to two years with a limit on the cumulative dose. It is interesting that even the small number of patients (only seven) who received therapy only for one year improved although the change in EDSS score was not significant.

The new emerging therapies viz, alemtuzumab, natalizumab, and autologous stem cell transplantation have also shown promising results with respect to improvement of disability. In a study by Cole et al., patients with RRMS showed an impressive reduction in disability at six months after Campath-1H (by a mean of 1.2 EDSS points) ${ }^{[25]}$ In addition, there was a further significant, albeit smaller, mean improvement in disability up to 36 months after treatment. ${ }^{[25]}$ In another study, Cole et al., recently reported a sustained reduction in disability in patients treated with alemtuzumab. ${ }^{[26]}$ Natalizumab also reduced the risk of disability progression by $64 \%$ and relapse rate by $81 \%$ in the treatment-naive patients with highly active disease and by $58 \%$ and $76 \%$, respectively, in patients with highly active disease despite IFNß-1a treatment ${ }^{[2]}$ In another multicentre study, the use of natalizumab was found to show significant improvement in the EDSS score ${ }^{[28]} \mathrm{A}$ phase I/II study in 21 patients using autologous nonmyeloablative haemopoietic stem cell transplantation in relapsing-remitting MS, showed significant improvements in neurological disability, as determined by EDSS score $(P<0.0001) \cdot{ }^{[29]}$ Immunoablation and autologous stem cell transplantation have also shown sustained EDSS benefits in some patients with aggressive MS. ${ }^{[30]}$

The common adverse events of mitoxantrone reported in the literature include leucopenia (10-19\%), nausea or vomiting $(18-85 \%)$, alopecia (33-61\%), urinary tract infections (6-32\%), and upper respiratory tract infections (4-53\%). ${ }^{[5]}$ A similar adverse event profile was evident in our study where four patients $(17 \%)$ developed leucopenia, two (9\%) developed a urinary tract infection, and five $(23 \%)$ reported mild hair loss. Patients treated with mitoxantrone are at increased risk for cardiac toxicity as manifested by reduced left ventricular ejection fraction, and congestive heart failure. ${ }^{[31]}$ Because of concerns about cardiac toxicity, a lifetime cumulative dose of mitoxantrone exceeding $140 \mathrm{mg} / \mathrm{m}^{2}$ is not recommended ${ }^{[5]}$ In our study, one patient with SPMS developed an asymptomatic cardiomyopathy that proved to be transient on follow-up cardiac ultrasound. This is similar to a study of 41 patients with SPMS in whom four of the seven patients with decreased EF showed posttreatment normalization of left ventricular EF (the other three patients were lost to follow-up). ${ }^{[32]}$ There is also a concern regarding TRAL. In the study by Ellis and Boggild the median onset of TRAL following mitoxantrone treatment was 18.5 months (range, 4-60 months). ${ }^{[6]}$ It is therefore necessary that the patients are followed up for at least five years.
This single-center study involved a small number of patients with no controls. Despite this limitation, we found that mitoxantrone, as an initial therapy, significantly reduced the clinical exacerbations and progression of disability associated with MS. In the short follow-up, the rate of serious adverse events was relatively low (two severe leucopenia and one cardiomyopathy), and the events were either transient or reversed with therapy. However, the patients require long-term follow-up for at least five years for delayed complications including TRAL. We believe that if monitored appropriately, mitoxantrone can be considered in the initial treatment of Indian patients with MS who cannot afford the more expensive first-line disease modifying treatments such as interferons and GA. A muilticentric trial involving a larger number of patients and long-term follow-up will be needed to assess the value of mitoxantrone therapy.

\section{Acknowledgments}

We are grateful to Dr. B. K. Goyal, Dean, Bombay Hospital Medical Institute of Medical Sciences for allowing us to use the medical records. We are grateful to Dr. Jayanthi Mani for helping in the statistical analysis.

\section{References}

1. Lublin FD, Reingold SC. Defining the clinical course of multiple sclerosis: Results of an international survey. National Multiple Sclerosis Society (USA) Advisory Committee on Clinical Trials of New Agents in Multiple Sclerosis. Neurology 1996;46:907-11.

2. Hartung HP, Gonsette R, König N, Kwiecinski H, Guseo A, Morrissey SP, et al. Mitoxantrone in Multiple Sclerosis Study Group (MIMS). Mitoxantrone in progressive multiple sclerosis: A placebo-controlled, double-blind, randomised, multicentre trial. Lancet 2002;360:2018-25.

3. Goodin DS, Arnason BG, Coyle PK, Frohman EM, Paty DW. Therapeutics and Technology Assessment Subcommittee of the American Academy of Neurology. The use of mitoxantrone (Novantrone) for the treatment of multiple sclerosis: Report of the Therapeutics and Technology Assessment Subcommittee of the American Academy of Neurology. Neurology 2003;61:1332-8.

4. Cohen BA, Khan O, Jeffery DR, Bashir K, Rizvi SA, Fox EJ, et al. Identifying and treating patients with suboptimal responses. Neurology 2004;63:S33-40.

5. Fox E.J. Management of worsening multiple sclerosis with mitoxantrone: A review. Clin Ther 2006;28:461-74.

6. Ellis R, Boggild M. Therapy-related acute leukaemia with Mitoxantrone: What is the risk and can we minimise it? Mult Scler 2009;15:505-8.

7. Martinelli V. Leukemia risk with Mitoxantrone for multiple sclerosis (abstract). American Academy of Neurology 61st Annual Meeting, 2009. LB3.001.

8. Poser CM, Paty DW, Scheinberg L, McDonald WI, Davis FA, Ebers GC, et al. New diagnostic criteria for multiple sclerosis: Guidelines for research protocols. Ann Neurol 1983;13:227-31.

9. Kurtzke JF. Rating neurologic impairment in multiple sclerosis: An expanded disability status scale (EDSS). Neurology 1983;33:1444-52.

10. Polman CH, Reingold SC, Edan G, Filippi M, Hartung HP, Kappos L, et al. Diagnostic criteria for multiple sclerosis: 2005 revisions to the "McDonald Criteria". Ann Neurol 2005;58:840-6.

11. MeDonald WI, Compston A, Edan G, Goodkin D, Hartung HP, 
Lublin FD, et al. Recommended diagnostic criteria for multiple sclerosis: Guidelines from the International Panel on the diagnosis of multiple sclerosis. Ann Neurol 2001;50:121-7.

12. Wingerchuk DM, Lennon VA, Pittock SJ, Lucchinetti CF, Weinshenker BG. Revised diagnostic criteria for neuromyelitis optica. Neurology 2006;66:1485-9.

13. Neuhaus O, Wiendl H, Kieseier BC, Archelos JJ, Hemmer B, Stüve O, et al. Multiple sclerosis: Mitoxantrone promotes differential effects on immunocompetent cells in vitro. J Neuroimmunol 2005;168:128-37.

14. Kopadze T, Dehmel T, Hartung HP, Stüve O, Kieseier BC. Inhibition by mitoxantrone of in vitro migration of immunocompetent cells: A possible mechanism for therapeutic efficacy in the treatment of multiple sclerosis. Arch Neurol 2006;63:1572-8.

15. Fox EJ. Mechanism of action of mitoxantrone. Neurology 2004;63:S15-8.

16. Rizvi SA, Zwibel H, Fox EJ. Mitoxantrone for multiple sclerosis in clinical practice. Neurology 2004;63:S25-7.

17. Touchette DR, Durgin TL, Wanke LA, Goodkin DE. A cost-utility analysis of mitoxantrone hydrochloride and interferon beta-1b in the treatment of patients with secondary progressive or progressive relapsing multiple sclerosis. Clin Ther 2003;25:611-34.

18. Buttinelli C, Clemenzi A, Borriello G, Denaro F, Pozzilli C, Fieschi C. Mitoxantrone treatment in multiple sclerosis: A 5-year clinical and MRI follow-up. Eur J Neurol 2007;14:1281-7.

19. Debouverie M, Taillandier L, Pittion-Vouyovitch S, Louis S, Vespignani H. Clinical follow-up of 304 patients with multiple sclerosis three years after mitoxantrone treatment. Mult Scler 2007;13:626-31.

20. Hamzehloo A, Etemadifar M. Mitoxantrone reduced disability in Iranian patients with multiple sclerosis. Arch Iran Med 2007;10:59-64.

21. Komori M, Tanaka M, Muramoto E, Ohno M, Matsumoto R, Murase N, et al. Mitoxantrone for the treatment of Japanese patients with multiple sclerosis. Rinsho Shinkeigaku 2007;47:401-6.

22. Weinstock-Guttman B, Ramanathan M, Lincoff N, Napoli SQ, Sharma J, Feichter J, et al. Study of mitoxantrone for the treatment of recurrent neuromyelitis optica (Devic disease). Arch Neurol 2006;63:957-63.

23. Vollmer T, Panitch H, Bar-Or A, Dunn J, Freedman MS, Gazda SK, et al. Glatiramer acetate after induction therapy with mitoxantrone in relapsing multiple sclerosis. Mult Scler 2008;14:663-70.
24. Ramtahal J, Jacob A, Das K, Boggild M. Sequential maintenance treatment with glatiramer acetate after mitoxantrone is safe and can limit exposure to immunosuppression in very active, relapsing remitting multiple sclerosis. J Neurol 2006;253:1160-4.

25. Coles AJ, Cox A, Le Page E, Jones J, Trip SA, Deans J, et al. The window of therapeutic opportunity in multiple sclerosis: Evidence from monoclonal antibody therapy. J Neurol 2006;253:98-108.

26. Coles AJ. CAMMMS223 Study Group. Alemtuzumab Induces a Sustained Reduction in Disability in Patients with Relapsing Remitting Multiple Sclerosis. American Academy of Neurology. 61st Annual Meeting, 2009. p. 145 .

27. Hutchinson M, Kappos L, Calabresi PA, Confavreux C, Giovannoni G, Galetta SL, et al. for the AFFIRM and SENTINEL Investigators. The efficacy of natalizumab in patients with relapsing multiple sclerosis: Subgroup analyses of AFFIRM and SENTINEL. J Neurol. 2009;256:405-15.

28. Putzki N, Yaldizli O, Mäurer M, Kuckert S, Cursiefen S, Tettenborn B, et al. Natalizumab Reduces Clinical and MRI Disease Activity in Second Line Treatment of Relapsing Remitting Multiple Sclerosis: Results of a Multicenter Study. American Academy of Neurology. $61^{\text {st }}$ Annual Meeting, 2009. p. 5-128.

29. Burt RK, Loh Y, Cohen B, Stefoski D, Balabanov R, Katsamakis G, et al. Autologous non-myeloablative haemopoietic stem cell transplantation in relapsing-remitting multiple sclerosis: A phase I/II study. Lancet Neurol 2009;8:244-53.

30. Mark S. Freedman, Harold L. Atkins, Marjorie Bowman, Canadian BMT Study Group. Neurological Recovery Following Treatment of Aggressive MS with Immunoablation and Autologous Stem Cell Transplantation. American Academy of Neurology, $61^{\text {st }}$ Annual Meeting, 2009. p 02.145 .

31. Ghalie RG, Edan G, Laurent M, Mauch E, Eisenman S, Hartung HP, et al. Cardiac adverse effects associated with mitoxantrone (Novantrone) therapy in patients with MS. Neurology 2002;59:909-13.

32. Peak S, Tsao-Wei DD, Chamberlain MC. Mitoxantrone in secondary progressive multiple sclerosis: A review of toxicity in 41 patients. Therapy $2007 ; 4: 55-58$

Accepted on 02-07-2009

Source of Support: Nil, Conflict of Interest: None declared. 